\title{
Sources of errors on visuoperceptual tasks: Role of education, literacy, and search strategy
}

\author{
Desiree A. Byrd ${ }^{\mathrm{a}}$, Diane M. Jacobs ${ }^{\mathrm{b}}$, H. John Hilton ${ }^{\mathrm{a}, \mathrm{c}, \mathrm{d}}$, Yaakov Stern ${ }^{\mathrm{a}, \mathrm{c}, \mathrm{d}}$, \\ Jennifer J. Manly,c,d,* \\ ${ }^{a}$ G. H. Sergievsky Center, Columbia University College of Physicians and Surgeons, New York, NY 10032, USA \\ ${ }^{\mathrm{b}}$ San Diego, CA, USA \\ ${ }^{\mathrm{c}}$ Cognitive Neuroscience Division, Taub Institute for Research on Alzheimer's Disease and the Aging Brain, \\ Columbia University College of Physicians and Surgeons, New York, USA \\ ${ }^{\mathrm{d}}$ Department of Neurology, Columbia University College of Physicians and Surgeons, New York, USA
}

Accepted 19 December 2004

Available online 28 January 2005

\begin{abstract}
The current study explored possible sources of demographic effects through analyses of errors from modified formats of the Benton Visual Retention Test (BVRT) completed by African American elders. Results indicate that: (1) reading level was a stronger predictor of BVRT performance than years of education; (2) on the single-item matching format of the task, individuals with lower reading levels disproportionately produced errors on items that differed in geometric, rather than spatial features; and (3) on a multiple-choice matching format, individuals with lower reading levels committed more errors on items where the target was located in the lower half of a $2 \times 2$ matrix. (c) 2005 Elsevier Inc. All rights reserved.
\end{abstract}

Keywords: Visuoperception; Demographic influences; Error analysis; Literacy

\section{Introduction}

Tests that utilize visual, as opposed to verbal, stimuli are commonly used in neuropsychological evaluations. These tests are especially useful for evaluating lateralized cognitive impairments as well as specific learning disabilities (Lezak, 1995). The Benton Visual Retention Test (BVRT; Benton, 1955, Benton, Hamsher, De, Varney, \& Spreen, 1983) is a commonly used visual test that assesses short-term/working memory, visuoperception, and visuoconstructional abilities. While the relationship between age, education, and BVRT performance is well documented, to date, few studies have attempted to identify the mechanisms that underlie this effect.

\footnotetext{
* Corresponding author. Fax: +1 2123052426

E-mail address: jjm71@columbia.edu (J.J. Manly).
}

The BVRT has been used internationally to detect and monitor neurodegenerative diseases such as Alzheimer's disease (Hasse-Sander, Horn, Mueller, \& Schroeder, 1996; Robinson-Whelen, 1992; Stern et al., 1992; Zappala, Measso, Cavarzeran, \& Grigoletto, 1995). Originally designed as a reproduction task, the BVRT's vast popularity is attributable, in part, to its alternate forms and varied administration formats (e.g., immediate reproduction after either a 5 or $10 \mathrm{~s}$ exposure period, delayed reproduction, or copy), which allow for repeat administrations with reduced practice effects. The BVRT, like most neuropsychological measures, is sensitive to a number of demographic factors. Numerous studies document decreased accuracy with increasing age and increased accuracy with higher levels of education (Coman et al., 1999, 2002; Resnick, Trotman, Kawas, \& Zonderman, 1995; Youngjohn, Larrabee, \& Crook, 1993). There is no evidence that gender is related 
to overall BVRT performance, after controlling for the influence of age and education (Coman et al., 1999). In an effort to better understand the relationship between education and BVRT performance, Le Carret et al. (2003) utilized a multiple-choice recognition format of the BVRT to determine whether the observed educational advantage was related to enhanced executive working memory abilities or improved visual discrimination among elders with higher levels of education. In a study with 829 non-demented participants, the authors found that elders with fewer years of education were disproportionately less accurate than those with higher education on items where the correct response appeared in the bottom half of a $2 \times 2$ matrix, suggesting that the influence of education on BVRT recognition performance is mediated by test taking skills involving an executive search strategy.

Though education level has been documented to be a powerful predictor of cognitive test performance, research by our group reveals that literacy and reading level are more accurate reflections of actual academic achievement than years of education (Manly et al., 1999; Manly, Jacobs, Touradji, Small, \& Stern, 2002). This is especially true for African American elders whose educational opportunities were limited due to historical factors such as segregation (Anderson, 1988). Available evidence demonstrates that the relative influence of reading level on cognitive test performance is stronger than the influence of years of education. Statistically controlling for reading level attenuates ethnicity related test performance differences for groups matched on years of education (Baird et al., 2004; Manly et al., 1999). Therefore, reading level, rather than years of education, is likely to provide greater insight into the sources of education- and ethnicityrelated performance variability on neuropsychological tests, including visual tasks such as the BVRT. Further, measures of reading achievement correlate with overall academic achievement (Wilkinson, 1993) and with direct measures of quality of education such as pupil expenditures, teacher/student ratios, and teacher education (Hedges, Laine, \& Greenwald, 1994). The current study uses the Reading Recognition subtest from the WRAT-3 (Wide Range Achievement Test-Version 3) as an estimate of quality of education, since this measure and other WRAT scores have been found to remain relatively stable over time (Johnstone \& Wilhelm, 1996; Wilkinson, 1993).

There is ample evidence documenting the impact of reading skill on non-reading related cognitive ability. Studies with international samples of adults and children demonstrate that literacy, independent of formal schooling, influences generalized cognition, and possibly cerebral organization such that differential processing styles and skills emerge in people with low or no literacy skills (Ostrosky-Solis, Ardila, Rosselli, Lopez-Arango, \& Uriel-Mendoza, 1998; Pontius, 1995, 1997; Rosselli, 1993; Rosselli \& Ardila, 2003). One consistent finding is that literacy affects the temporal and spatial processing of visual, non-lettered information (Ardila, Rosselli, \& Rosas, 1989; Au \& Lovegrove, 2001; Conlon, Sanders, \& Zapart, 2004; Matute, Leal, Zarabozo, Robles, \& Cedillo, 2000; Reis, Guerreiro, \& Castro-Caldas, 1994). Therefore, it is possible that the impact of reading level on BVRT performance is mediated through differences in the perceptual processing of test items that vary in spatial and geometric characteristics. The present study evaluated this association by examining the ability of elders with a wide range of reading skills to accurately match BVRT items that differ with respect to shape and order.

In addition to the education effect, ethnicity also influences performance on the BVRT. We have found that non-demented African American and Hispanic elders achieve significantly lower scores than Caucasians on the recognition format of the BVRT (Manly et al., 1999, 2002; Stern et al., 1992; Stricks, Pittman, Jacobs, Sano, \& Stern, 1998). However, the mechanism for this effect is not yet known. Detailed qualitative analysis of errors made during the BVRT may shed light on the underlying causes of ethnic differences by identifying specific mechanisms responsible for lower scores. Prior studies of demographic influences on the BVRT were either completed in exclusively Caucasian samples (Resnick et al., 1995; Robinson-Whelen, 1992) or the ethnicity of the samples was not reported (Coman et al., 2002). Therefore, the influence of basic demographic variables on BVRT performance in African Americans remains unclear.

The current study utilized delayed recognition and matching formats of the BVRT to identify specific sources of performance variance in an elderly African American sample. BVRT stimuli were chosen due to the geometric simplicity of the figures and the documented differences on this test between elders of different ethnic groups (Stern et al., 1992). Further, the recognition format of testing, relative to the drawing response format, eliminates the influence of visuomotor and manual dexterity problems common in the elderly (Benton et al., 1983). We wanted to determine which stimulus features participants used to distinguish test items and whether the use of these features varied with reading level. The following hypotheses were tested: (1) reading level will emerge as a stronger predictor of BVRT performance than years of education; (2) elders with lower reading levels will demonstrate a unique pattern of perceptual processing by failing to successfully discriminate stimuli that differ in both spatial and geometric characteristics; and (3) consistent with results from Le Carret et al. (2003), individuals with lower reading levels will demonstrate less effective response search strategy by producing fewer successful responses to target items appearing in the lower half of $2 \times 2$ response matrices. 


\section{Methods}

\subsection{Research participants}

The study sample was comprised of 100 non-demented African American participants in the Washington Heights-Inwood Columbia Aging Project (WHICAP), a community-based, epidemiological study of dementia in Northern Manhattan, New York. The WHICAP study follows a random sample of elderly who received or were eligible for Medicare and resided in selected census tracts of Washington Heights and Inwood. The mean age of the participant group was 75.5 years $(S D=6.1$, range $=65$ 91) and all elders spoke English as their primary language. Ethnicity was determined by self-report following US Census criteria (United States Office of Management and Budget, 1997). Only those participants who identified themselves as non-Hispanic Black were eligible for the current study. The self-reported education level of the group was 12.5 years $(S D=3.4$, range $=5-20$ years). The sample was $77 \%$ female.

\subsection{Inclusion/exclusion criteria}

Potential participants were excluded if they had a history of Parkinson's disease, stroke, alcohol abuse, or serious mental illness such as depression or schizophrenia. Further, only those participants who exhibited no neurological or functional signs of dementia were included in the study. This determination was made on the basis of a clinical examination by our research physicians, which included a cognitive screening exam (Blessed, Tomlinson, \& Roth, 1968) and ratings of daily functioning (Schwab, England, Gillingham, \& Donaldson, 1969). The research physician's diagnosis was used as a "gold standard" for the absence of dementia, since the physician's assessment was made independent of the participant's performance on the neuropsychological battery. Finally, participants who experienced difficulty viewing the BVRT stimuli were excluded.

\subsection{Measures}

The standard 10-item multiple-choice delayed recognition format of the BVRT, as well as two experimental tasks based on BVRT stimuli and procedures, were utilized in this study. The experimental formats were created to allow for more detailed exploration of the sources of errors on the standard BVRT.

\subsection{Multiple-choice matching format BVRT}

For this experimental version of the task, participants were simultaneously presented with a single figure from the BVRT (Form C) and a multiple-choice array of three distractors and one target item in a $2 \times 2$ matrix. The seven target items chosen for this task were items that demonstrated the greatest ethnicity effects in a prior item analysis of responses to the BVRT delayed recognition format (Jacobs, personal communication, October, 2003). The seven items, two of which contained single figures and five were multi-figured, were presented over 15 trials. The four items that contained the most complex arrangement of figures were repeated three times throughout the test, each time with a different set of distractors. Participants were given the following instructions "See this shape up here (point)? See these four shapes down here? One of these four small shapes is the same as this one up here (point). Which of these four shapes down here is the same as this one up here?" No practice trials were administered. Correct scores were calculated as the number of correctly matched targets. The correct answer appeared in quadrant $\mathrm{A}$ in three trials, quadrant $\mathrm{B}$ in four trials, quadrant $\mathrm{C}$ in three trials, and quadrant $\mathrm{D}$ in five trials.

\subsection{Single-item matching BVRT}

A forced-choice, single item visuoperception task was experimentally developed by one of the authors (D.J.) to assess participants' ability to accurately discriminate same and non-same figures. The single item format consisted of the seven target items from the matching test described above. The items were presented over a total of 73 trials in one of three conditions: figure was identical to target $(n=22)$, figure differed from target with respect to shape of figure [shape distractors, $(n=29)$ ], and figure differed from target with respect to spatial arrangement/order of shapes [order distractors, $(n=22)$ ]. Participants were read the following instructions: "I want you to tell me if the figures down here (point) are exactly the same as the figures here (point to target). To be exactly the same, it has to be all of the same figures, oriented in the same direction, and presented in the same order." A learning trial of three example items was administered, during which feedback was provided (reinforcement or explanation and correction, if necessary). Feedback was not provided during the actual test. If participants failed to learn the task during the sample trials, test items were not administered. Responses to each of the single-item matching trials were a verbal reply of "Yes" or "No."

\subsection{Multiple-choice delayed recognition format BVRT}

Participants were shown a target stimulus from Form D of the BVRT (Benton, 1955) for $10 \mathrm{~s}$, after which it was removed from sight. The participant was then asked to identify, from memory, the target design from a fourchoice array presented in a $2 \times 2$ matrix. All stimuli were two-dimensional line drawings of familiar geometric shapes (i.e., circles, rectangles, triangles, etc.). Target 
stimuli were the same size as the original stimuli and were presented on $8.5 \times 11$ paper; the size of the response stimuli was $25 \%$ of the original. This test consisted of 10 items. The test instructions were, "Now I am going to show you a design for $10 \mathrm{~s}$ and then I am going to remove it and ask you to recall it from memory. I will ask you to choose the one that is the exact match. Look carefully so that you will be able to remember the design. Which one looks exactly like the one you just saw?" Total score was calculated as the total number of correctly identified items. Participants' responded by pointing to the desired response box. No response time limit was imposed.

\subsection{Reading skill}

Reading skill was measured using the Reading Recognition subtest from the Wide Range Achievement Test-Version 3 (WRAT-3; Wilkinson, 1993), which required participants to name letters and pronounce words out of context. The words were listed in order of decreasing familiarity and increasing phonological complexity. Consistent with the standard administration, a basal of five correct items and a ceiling of 10 incorrect items was used. Raw scores were converted to grade equivalent scores that reflect grade year and month, derived from the WRAT-3 normative sample of people aged 65-74 years. The lowest possible grade and month equivalent was 4.1 while the highest equivalent was postHigh School.

\subsection{Statistical procedures}

Separate simultaneous multiple regression analyses, with age, years of education, and reading scores as predictors, were used to evaluate the relative importance of these variables to performance on the BVRT recognition and matching tests. To investigate the impact of reading levels on error type, participants were categorized into tertile groups (low, medium, and high) based upon the distribution scores on the WRAT-3. ANOVA and $\chi^{2}$ analyses were used to compare age, years of education, and gender compositions of the reading groups. Group differences in error type were evaluated using MANCOVA, with age, and years of education as covariates.

\section{Results}

\subsection{Reading level vs years of education}

To demonstrate the extent of the discrepancy between years of education and reading level, the frequency of elders who earned grade equivalent reading scores at least one full year above or below their reported years of education was computed. Forty-four percent of elders earned reading scores that were below their years of education, $29 \%$ earned scores that corresponded to their completed educational level, and $27 \%$ earned scores at least one year above their reported educational level.

\subsection{Demographic predictors of BVRT matching and recognition performance}

Three separate multiple regression analyses were performed for the BVRT multiple-choice matching, singleitem matching, and recognition formats. Age, years of education, and reading level were simultaneously entered into each regression equation. Reading skill emerged as the single significant predictor of performance for the multiple-choice matching $(\beta=.39, p<.01)$, single-item matching $(\beta=.43, p<.01)$, and delayed recognition tests $(\beta=.51, p<.01)$. Neither age nor years of education entered the regression equation as contributing independent predictive value to test performances at the .05 level. $\beta$ Values for all predictor variables are presented in Table 1.

\subsection{Reading level and error type}

Readers were grouped into high, medium, and low groups based upon WRAT-3 performance (see Section 2 ). Elders in the high reading level group were significantly younger than elders in the medium reading level group $(F(2,92)=4.25, p<.05)$. As expected, the low reading level group had completed significantly fewer years of education than the medium and high reading level groups $(F(2,92)=7.30, p<.01)$. Gender compositions did not differ significantly among the reading groups $(p>.05)$. The BVRT single item matching format was used to examine the frequency of specific error types in each group. A 3 (reading level) $\times 3$ (test condition) MANCOVA, with years of education and age as covariates, was used to compare the performance of the reading groups on accuracy (hits and correct rejections) within the three conditions. Results reveal an omnibus effect of reading level $(F(6,172)=2.99, p=.008)$. As presented in Table 2, univariate follow-up tests indicate that the low reading group made significantly more errors than the medium and high level reading groups on items in the shape condition, in which the response item differed from the target with respect to shape of the figure $(p<.01)$. Reading level did not have a statistically

Table 1

Demographic predictors of BVRT performance: multiple regression

\begin{tabular}{lllll}
\hline & $\begin{array}{l}\text { Adjusted } \\
R^{2}\end{array}$ & $\begin{array}{l}\text { Age } \\
(\beta)\end{array}$ & $\begin{array}{l}\text { Education } \\
(\beta)\end{array}$ & $\begin{array}{l}\text { Reading level } \\
(\beta)\end{array}$ \\
\hline Multiple-choice matching & .14 & -.10 & -.01 & $.39^{*}$ \\
Single-item matching & .16 & -.02 & -.01 & $.43^{*}$ \\
Recognition & .33 & -.12 & .09 & $.51^{*}$ \\
\hline
\end{tabular}

* $p<.01$. 
Table 2

Reading level and accuracy on single-item matching BVRT by condition

\begin{tabular}{llll}
\hline & $\begin{array}{l}\text { Low reading } \\
\text { level }(n=32) \\
M(S D)\end{array}$ & $\begin{array}{l}\text { Medium reading } \\
\text { level }(n=33) \\
M(S D)\end{array}$ & $\begin{array}{l}\text { High reading } \\
\text { level }(n=29) \\
M(S D)\end{array}$ \\
\hline $\begin{array}{l}\text { Same }(\max =22) \\
\begin{array}{c}\text { Different order } \\
(\max =22)\end{array}\end{array}$ & $21.3(1.1)$ & $21.5(.91)$ & $21.5(1.4)$ \\
$\begin{array}{c}\text { Different shape** } \\
(\max =29)\end{array}$ & $26.6(2.6)$ & $21.8(.44)$ & $21.6(1.7)$ \\
\hline$* * p<.01$. & & & $28.7(.54)$ \\
& & &
\end{tabular}

Table 3

BVRT Multiple-choice recognition: proportionate accuracy by response location

\begin{tabular}{|c|c|c|c|c|c|}
\hline \multicolumn{2}{|c|}{$\begin{array}{l}\text { Low reading } \\
\text { level }(n=32)\end{array}$} & \multicolumn{2}{|c|}{$\begin{array}{l}\text { Medium reading } \\
\text { level }(n=24)\end{array}$} & \multicolumn{2}{|c|}{$\begin{array}{l}\text { High reading } \\
\text { level }(n=24)\end{array}$} \\
\hline $93 \%$ & $91 \%$ & $96 \%$ & $93 \%$ & $100 \%$ & $94 \%$ \\
\hline $81 \%$ & $77 \%$ & $93 \%$ & $94 \%$ & $94 \%$ & $97 \%$ \\
\hline
\end{tabular}

significant effect on performances on the other conditions $(p>.05)$.

\subsection{Reading level and error location}

Elders in the low reading group earned significantly lower scores on the BVRT multiple-choice matching format than the medium and high reading groups $(F(2,91)=11.9, p<.01)$, which did not differ from one another. As a qualitative exploration of the source of this difference, accuracy in each of the response quadrants was examined within each reading level group. Accuracy was defined as the proportion of correct answers in each quadrant. Proportionate, rather than raw values were used for analyses because the location of the target figure among the distractors was not evenly dispersed throughout the quadrants. The low literacy group was less successful at detecting targets when they appeared in the lower two quadrants (average success rate for lower quadrants $=79 \%$, upper quadrants $=92 \%$ ). The medium and high literacy groups demonstrated practically identical success rates in the quadrants (medium group: average success rate for lower quadrants $=94 \%$, upper quadrants $=95 \%$; high group: lower quadrants $=95 \%$, upper quadrants $=99 \%$; see Table 3 ).

\section{Discussion}

The current study explored the relationship between age, education, reading level, and performance on tests of visuoperception (modified formats of the BVRT). Given reported differences between African American and Caucasian elderly on the BVRT (Manly et al., 2002;
Stern et al., 1992), this study was completed with an African American sample to identify possible sources of previously reported, relatively lower group performance on this test.

Consistent with prior reports of the augmented importance of reading level over years of education (Manly et al., 1999), results from this study indicate that reading level is a better predictor of BVRT matching and delayed recognition performance than years of education. When age, education years, and reading level were evaluated for their BVRT predictive value, reading level emerged as the sole significant predictor for both the matching and recognition formats of the test. This result demonstrates the importance of reading level to non-verbal/visual tests and suggests that reading level, more so than education level, should be considered when estimating expected cognitive test performance. This is especially true among ethnic minorities, who, because of historical disparities in educational quality, often demonstrate significant discrepancies in the quantity and quality of their educational experiences. The impact of unequal educational quality on achievement, test performance and other outcomes (wage earnings) in African Americans is well documented (Baker, Johnson, Velli, \& Wiley, 1996; Hanushek, 1989; Margo, 1985). Importantly, $44 \%$ of our sample were currently reading at levels that were more than one year below their reported years of school and are at increased risk for false positive diagnoses. Likewise, $27 \%$ of the sample had reading levels that were higher than their reported years of education and are equally at risk for false negative diagnosis given that performance expectations may be lower than their true baseline ability. In all, results from this study support the use of reading level, rather than completed years of education, to establish premorbid performance expectations and to assist with neurocognitive diagnoses.

Since the delayed recognition format of the BVRT is somewhat complex, we developed a single-item matching format using BVRT stimuli to determine the specific conditions under which errors are made. The presence of any differences on this task is noteworthy because this simplified version contained significantly decreased cognitive demands; elders had to decide whether two basic geometric figures, presented simultaneously, were exactly the same. Differences between the target and distractor items were not subtle but were largely apparent (i.e., a square versus a circle). Results reveal that elders with lower reading levels made more errors on items that differed in geometric properties (i.e., a circle versus a square) but not those with different spatial arrangements (i.e., circle-triangle-circle versus triangle-circle-circle). The specificity of this response bias is further evidenced by the absence of group differences in the ability to accurately identify matching figures that differed in the spatial arrangement of the shapes. Since elders with lower 
reading levels did not demonstrate difficulty using spatial arrangement details to determine sameness, it appears that increased reading level is associated with enhanced accurate perception or salience of shapes. Evidence from other studies supports this notion. Matute et al. (2000), in their examination of illiterate, semi-literate, and literate adults, report that errors of global fidelity (inability to holistically recreate a model) in a stick figure test, were more discriminative of the literacy groups than errors of spatial rotation or distortion, suggesting a specific perceptual difference between the groups. Additionally, Pontius (1997) compared schooled literate to unschooled illiterate Ethiopian adolescents on their ability to complete the Kohs Block Design test and reported that illiterates' were able to accurately reproduce the overall configuration of the designs yet their errors on the task generally neglected internal geometric shapes present in the target designs. In all, the source of the literacy effect on visual discrimination for geometric shapes appears to be related to variation in geometric perception rather than generalized decreased spatial ability.

As hypothesized, reading level was related to search strategy on the BVRT multiple- choice matching test. Elders with lower reading levels were less accurate when the correct response appeared on the bottom half of the $2 \times 2$ response display. These findings replicate those from the study by Le Carret et al. (2003), where even larger discrepancies were observed in the search strategy of French elders with different educational levels on a similar task. Differences between our findings and those of Le Carret et al. (2003) may be attributable to differences in sample sizes as well as differences in the distribution of educational/reading levels. Also, the former study employed a 10-item multiple-choice matching test while our study used a 15 -item test. Despite the differences in the magnitude of findings, the pattern of results is similar. One possible explanation for the differential search strategies by reading level, as Le Carret et al. (2003) propose, is that increased reading or educational level enhances the executive control component of working memory, resulting in elders with more education or higher reading skill, to demonstrate more a test taking skill that includes searching response displays more thoroughly, which is a more efficient test taking strategy. Our working model of the relationship between reading level and performance on measures such as the BVRT uses as its basis a model of the working memory system proposed by Engle, Tuholski, Laughlin, and Conway (1999). In this model, heuristics, or search strategies and skills such as grouping, rehearsal, and coding are hypothesized to be involved in structuring and maintaining memory traces. We hypothesize that the development of literacy skills greatly aids in the acquisition of heuristics and thus, better test taking strategies. The degree to which heuris- tics have become automatic and routine determines whether executive attention is required to maintain those memory traces. We propose that among adults with high reading levels, heuristics are developed to the point where a task such as the BVRT may be largely routine and involve only minimal memory and visual processing resources. Among low literacy individuals with poorly developed heuristics, meeting the task requirements of the BVRT may require additional allocation of attention by the central executive (Van den Broek, Young, Tzengy, \& Linderholm, 1999). A study by Ostrosky-Solis, Efron, and Yund (1991) supports the assertion that reading level influences search strategies on a visual task involving non-verbal stimuli. These investigators found that the sequence of visual scan on a non-verbal target detection task that involved discrimination of patterns was more consistent across literate subjects than among illiterate subjects. In other words, there was more variability in the scanning sequence used by illiterates than among literates, which could further contribute to less developed search strategies and test taking skills. Another possible explanation for this pattern of results is that individuals with lower reading levels are more likely to mistakenly choose one of the first shapes attended to, thus discontinuing their search of all available options. Additional research is necessary to test these possible explanations.

The current study, through the use of qualitative and quantitative methods, reveals that reading level is an important reflection of mechanisms responsible for variation in performance on visuoperceptual tests. Elders with lower reading levels made disproportionately more errors on items that differed in simple geometric shape during a single-item matching task and demonstrated a differential visual search pattern than elders with higher reading levels. The current results provide several directions for future research. It will be useful to characterize visuoperceptual performance patterns in the extreme case of illiteracy such as those with minimal or no reading or writing skills. Additional research with carefully designed experiments is necessary to isolate search strategy from generalized poor shape recognition skills. For example, it would be informative for future studies to record eye movements (Rayner, 1998) and timed responses to multiple-choice, visual search tasks using verbal, and non-verbal stimuli (such as the Sternberg paradigm) to examine the relationship between literacy and the cognitive processes that underlie performance on more complex visuoperception tasks. Also replication of these findings with larger and more ethnically diverse samples using varied visual stimuli is necessary before the present results can be generalized. Although our study was completed with an entirely African American sample, we expect these patterns of results to apply to other ethnicity groups with varied reading levels. 


\section{References}

Anderson, J. D. (1988). The education of Blacks in the South. Chapel Hill: University of North Carolina Press pp. 1860-1935.

Ardila, A., Rosselli, M., \& Rosas, P. (1989). Neuropsychological assessment in illiterates: Visuospatial and memory abilities. Brain and Cognition, 11, 147-166.

Au, A., \& Lovegrove, B. (2001). Temporal processing ability in above average and average readers. Perception and Psychophysics, 63, 148-155.

Baird, R., Mindt, M., Ryan, E., Monzones, J., Naseer, M., \& Morgello, S. (2004). The utility of norms based on education versus reading ability in the prediction of functional impairment in HIV-infection. Poster session Presented at the Annual Meeting of the International Neuropsychological Society, Baltimore, MD.

Baker, F. M., Johnson, J. T., Velli, S. A., \& Wiley, C. (1996). Congruence between education and reading levels of older persons. Psychiatric Services, 47, 194-196.

Benton, A. L. (1955). The visual retention test. New York, NY: The Psychological Corporation.

Benton, A. L., Hamsher, K., De, S., Varney, N., \& Spreen, O. (1983). Contributions to neuropsychological assessment: A clinical manual. New York, NY: Oxford University Press.

Blessed, G., Tomlinson, B. E., \& Roth, M. (1968). The association between quantitative measures of senile change in the cerebral grey matter of elderly subjects. British Journal of Psychology, 114, 797-811.

Coman, E., Moses, J., Kraemer, H., Friedman, L., Benton, A., \& Yesavage, J. (1999). Geriatric performance on the Benton visual retention test: Demographic and diagnostic considerations. Clinical Neuropsychologist, 13, 66-77.

Coman, E., Moses, J., Kraemer, H., Friedman, L., Benton, A., \& Yesavage, J. (2002). Interactive influences on BVRT performance level: Geriatric considerations. Archives of Clinical Neuropsychology, 17, 595-610.

Conlon, E., Sanders, M., \& Zapart, S. (2004). Temporal processing in poor adult readers. Neuropsychologia, 42, 142-157.

Engle, R. W., Tuholski, S. W., Laughlin, J. E., \& Conway, A. R. (1999). Working memory, short-term memory and general fluid intelligence: A latent variable approach. Journal of Experimental Psychology: General, 3, 309-331.

Hanushek, E. (1989). The impact of differential expenditures on school performance. Educational Researcher, 18, 45-51.

Hasse-Sander, I., Horn, R., Mueller, H., \& Schroeder, M. R. (1996). Validity of the Benton test in the diagnosis of Alzheimer's disease. Zeitshcrift fur Gerontopsychologie \& -P sychiatrie, 9, 65-77.

Hedges, L. V., Laine, R. D., \& Greenwald, R. (1994). Does money matter? A meta-analysis of studies of the effects of differential school inputs on student outcomes. Educational Researcher, 23, 5-14.

Johnstone, B., \& Wilhelm, K. L. (1996). The longitudinal stability of the WRAT-R reading subtest: Is it an appropriate estimate of premorbid intelligence? Journal of the International Neuropsychological Society, 2, 282-285.

Le Carret, N., Rainville, C., Lechevallier, N., Lafont, S., Letenneur, L., \& Fabrigoule, C. (2003). Influence of education on the benton visual retention test performance as mediated by a strategic search component. Brain and Cognition, 53, 408-411.

Lezak, M. D. (1995). Neuropsychological assessment (3rd ed). New York: Oxford University Press.

Manly, J. J., Jacobs, D. M., Sano, M., Bell, K., Merchant, C. A., Small, S. A., et al. (1999). Effect of literacy on neuropsychological test performance in nondemented, education-matched elders. Journal of the International Neuropsychological Society, 5, 191-202.

Manly, J. J., Jacobs, D. M., Touradji, P., Small, S. A., \& Stern, Y. (2002). Reading level attenuates differences in neuropsychological test performance between African American and White elders. Journal of the International Neuropsychological Society, 8, 341348.
Margo, R. A. (1985). Disenfranchisement, school finance, and the economics of segregated schools in the United States south. New York: Garland Publishing pp. 1980-1910.

Matute, E., Leal, F., Zarabozo, D., Robles, A., \& Cedillo, C. (2000). Does literacy have an effect on stick construction tasks?. Journal of the International Neuropsychological Society, 6, 668-672.

Ostrosky-Solis, F., Efron, R., \& Yund, E. (1991). Visual detectability gradients: Effect of illiteracy. Brain and Cognition, 17, 42-51.

Ostrosky-Solis, F., Ardila, A., Rosselli, M., Lopez-Arango, G., \& UrielMendoza, V. (1998). Neuropsychological test performance in illiterate subjects. Archives of Clinical Neuropsychology, 13, 645-660.

Pontius, A. (1995). In similarity judgments hunter-gatherers prefer shapes over spatial relations in contrast to literate groups. Perceptual and Motor Skills, 83, 1027-1041.

Pontius, A. (1997). Impact of literacy training on spatial representations in Southwest Ethiopia. International Journal of Intercultural Relations, 21, 299-304.

Rayner, K. (1998). Eye movements in reading and information processing: 20 years of research. Psychological Bulletin, 124, 372-422.

Reis, A., Guerreiro, M., \& Castro-Caldas, A. (1994). Influence of educational level of non brain-damaged subjects on visual naming capacities. Journal of Clinical and Experimental Neuropsychology, 16, 939-942.

Resnick, S., Trotman, K., Kawas, C., \& Zonderman, A. (1995). Ageassociated changes in specific errors on the Benton Visual Retention Test. Journal of Gerontology B, 50, 171-178.

Robinson-Whelen, S. (1992). Benton Visual Retention Test performance among normal and demented older adults. Neuropsychology, 6, 261-269.

Rosselli, M. (1993). Neuropsychology of illiteracy. Special Issue: The origins of cognitive activity. Behavioural Neurology, 6, 107-112.

Rosselli, M., \& Ardila, A. (2003). The impact of culture and education on non-verbal neuropsychological measurements: A critical review. Brain and Cognition, 52, 326-333.

Schwab, J. F., England, A. C., Gillingham, F. H., \& Donaldson, M. C. (1969). Projection technique for evaluating surgery in Parkinson's disease. In Third symposium on Parkinson's disease (pp. 152-157). New York, NY: Churchill Livingstone Inc.

Stern, Y., Andrews, H., Pittman, J., Sano, M., Tatemichi, T., Lantigua, R., et al. (1992). Diagnosis of dementia in a heterogeneous population. Development of a neuropsychological paradigm-based diagnosis of dementia and quantified correction for the effects of education. Archives of Neurology, 49, 453-460.

Stricks, L., Pittman, J., Jacobs, D., Sano, M., \& Stern, Y. (1998). Normative data for a brief neuropsychological battery administered to English- and Spanish-speaking community-dwelling elders. Journal of the International Neuropsychological Society, 4, 311318.

United States Office of Management and Budget, (1997). Revisions to the Standards for the Classification of Federal Data on Race and Ethnicity (October 30, 1997). Standards for maintaining, collecting, and presenting federal data on race and ethnicity. United States Office of Management and Budget [On-line]. Available: http:// www.whitehouse.gov/omb/fedreg/ombdir15.html.

Van den Broek, H., Young, M., Tzengy, Y., \& Linderholm, T. (1999). The landscape model of reading: Inferences and the online construction of a memory representation. In H. van den Oostendorp \& S. R. Goldman (Eds.), The construction of mental representations during reading (pp. 71-98). Erlbaum, $\mathrm{NJ}$ : Mawhah.

Wilkinson, G. S. (1993). Wide Range Achievement Test, 1993 edition.

Youngjohn, J. R., Larrabee, G. J., \& Crook, T. H. (1993). New adult age- and education-correction norms for the Benton Visual Retention Test. Clinical Neuropsychologist, 7, 155-160.

Zappala, G., Measso, G., Cavarzeran, F., \& Grigoletto, F. (1995). Aging and memory: Corrections for age, sex and education for three widely used memory tests. Italian Journal of Neurological Sciences, $16,177-184$. 